\title{
Differentiation of pulmonary complications with extensive ground-glass attenuation on high-resolution CT in immunocompromised patients
}

\author{
Yoshie Kunihiro $^{1}\left(\right.$ Nobuyuki Tanaka $^{2} \cdot$ Reo Kawano $^{3} \cdot$ Tsuneo Matsumoto $^{2} \cdot$ Taiga Kobayashi $^{2} \cdot$ Toshiaki Yujiri $^{4}$. \\ Makoto Kubo ${ }^{5} \cdot$ Toshikazu Gondo $^{6} \cdot$ Katsuyoshi Ito ${ }^{1}$
}

Received: 23 January 2021 / Accepted: 10 April 2021 / Published online: 4 May 2021

(c) The Author(s) 2021

\begin{abstract}
Purpose The purpose of this study was to compare the high-resolution CT (HRCT) findings of pulmonary infectious and noninfectious complications with extensive ground-glass attenuation (GGA) in immunocompromised patients.

Materials and methods One hundred fifty-two immunocompromised patients with pulmonary complications that showed extensive GGA ( $>50 \%$ of the whole lung on HRCT) were included in this study. The diagnoses of the 152 patients were as follows: pneumocystis pneumonia (PCP), $n=82$; drug-induced pneumonia, $n=38$; bacterial pneumonia, $n=9$; cytomegalovirus pneumonia, $n=6$; idiopathic pneumonia syndrome, $n=6$; diffuse alveolar hemorrhage (DAH), $n=4$; fungal infection, $n=3$; tuberculosis, $n=2$ and pulmonary edema, $n=2$. Two chest radiologists retrospectively evaluated the CT criteria, which consisted of 12 findings.

Results The nodule $(p=0.015)$, the bronchovascular bundle (BVB) thickening $(p=0.001)$, and the interlobular septum (ILS) thickening $(p=0.002)$ were significantly infrequent in PCP. The ILS thickening was significantly frequent in drug-induced pneumonia $(p<0.001)$ though it was also frequent in other noninfectious and infectious diseases. The BVB thickening was significantly frequent in bacterial pneumonia $(p=0.005)$. The nodule was significantly frequent in DAH $(p=0.049)$.

Conclusion Nodules, BVB thickening, and ILS thickening could be useful HRCT findings for the differential diagnosis of pulmonary complications in immunocompromised patients with extensive GGA.
\end{abstract}

Keywords X-ray computed tomography $\cdot$ Ground-glass opacity $\cdot$ Immunocompromised host $\cdot$ Multivariate analysis

\section{Introduction}

Ground-glass attenuation (GGA) or ground-glass opacity (GGO) is defined as increased attenuation of the lung parenchyma without obscuration of the pulmonary vessels on high-resolution CT (HRCT) and is caused by a wide

Yoshie Kunihiro

kyoshie@yamaguchi-u.ac.jp

1 Present Address: Department of Radiology, Yamaguchi University Graduate School of Medicine, 1-1-1

Minamikogushi, Ube, Yamaguchi 755-8505, Japan

2 Department of Radiology, National Hospital Organization Yamaguchi - Ube Medical Center, 685 Higashikiwa, Ube, Yamaguchi 755-0241, Japan

3 Center for Integrated Medical Research, Hiroshima University Hospital, Kasumi 1-2-3 Minami-ku, Hiroshima 734-8551, Japan variety of interstitial and alveolar diseases [1, 2]. In immunocompromised patients, pulmonary complications, especially those which show extensive GGA on HRCT, could be major causes of morbidity and mortality in immunocompromised patients. Acute or subacute diffuse GGA may be caused by opportunistic infections, such as pneumocystis

4 Division of Endocrinology, Metabolism, Hematological Science and Therapeutics, Yamaguchi University Graduate School of Medicine, 1-1-1 Minamikogushi, Ube, Yamaguchi 755-8505, Japan

5 Department of Medicine and Clinical Science, Yamaguchi University Graduate School of Medicine, 1-1-1 Minamikogushi, Ube, Yamaguchi 755-8505, Japan

6 Division of Pathology, Fujisawa City Hospital, 2-6-1 Fujisawa, Fujisawa, Kanagawa 251-8550, Japan 
pneumonia (PCP), cytomegalovirus pneumonia (CMV-P), and noninfectious diseases, including drug-induced pneumonia, pulmonary edema, diffuse alveolar hemorrhage (DAH), and acute respiratory distress syndrome (ARDS) [3]. The underlying causes of ARDS could include sepsis, pneumonia, and other infectious or noninfectious conditions. ARDS shows diffuse alveolar damage (DAD) pattern on histology. In patients treated with hematopoietic stem cell transplantation (HSCT), idiopathic pneumonia syndrome (IPS) could also occur as diffuse lung injury $[4,5]$. It is important to distinguish between these diseases because the therapeutic strategies are different; however, it is challenging due to the similarities of the clinical and HRCT findings.

The objective of this study was to evaluate and compare the HRCT findings of pulmonary complications with extensive GGA on HRCT in immunocompromised patients and identify indicators that can be used for differentiation between these complications.

\section{Materials and methods}

This study was approved by the institutional review board of our hospital, and the requirement for written informed consent was waived because of the retrospective design.

\section{Patients}

We retrospectively reviewed the $\mathrm{CT}$ images of immunocompromised patients with acute chest complications at our hospital from January 1990 to December 2015. Immunocompromised state was defined as having diseases or conditions which cause immunosuppression, or taking immunosuppressive treatment. The selection criteria for this study were as follows: (1) the patients underwent HRCT scans, (2) HRCT showed extensive GGA ( $>50 \%$ of the whole lung), and (3) only one final diagnosis (with the exception of tumor infiltration) was made for each patient. At first, we identified a total of 1073 patients. Among them, 478 patients were excluded because the lung diseases were not specified in spite of the detailed evaluation of medical records and laboratory findings, including sputum culture, serological tests for several organisms, or the results of bronchoalveolar lavage (BAL), transbronchial lung biopsy (TBLB), blood culture, urinary antigen and autopsy. ARDS cases were also excluded because the causes could be overlapping and they were difficult to specify. Among infectious diseases, 25 patients were excluded because of the existence of co-infections. Then, 83 patients with pulmonary infiltration of tumor (underlying disease) were excluded because it could be difficult to specify the cause of diffuse GGA and exclude other noninfectious or infectious complications in cases who already had pulmonary involvement by malignancy. In addition, 11 patients in whom complications occurred within four weeks after a previous episode were excluded from this study because the previous episode might have influenced the HRCT findings of the current disease. Finally, 324 patients without extensive GGA were excluded. One hundred fiftytwo patients ( 83 males and 69 females; mean age ( \pm standard deviation [SD]): $59.6 \pm 16.1$ years; range: $3-90$ years) were included in this study. Among these, 4 patients had 2 episodes. The final diagnosis of the 152 patients were as follows: PCP $(n=82)$, drug-induced pneumonia $(n=38)$, bacterial pneumonia $(n=9)$, CMV-P $(n=6)$, IPS $(n=6)$, DAH $(n=4)$, fungal infection $(n=3)$, TB $(n=2)$, and pulmonary edema $(n=2)$. Table 1 shows the underlying diseases and the diagnostic methods of all patients (Table 1). Some cases were diagnosed based on the clinical course and these diagnoses were basically determined by physicians according to the detailed analysis of all physical and laboratory findings and responsiveness to therapy. The criteria for the diagnoses according to the clinical course were as follows.

Drug-induced pneumonia: (1) onset of lung diseases after chemotherapy; (2) immediate responsiveness to newly administrated steroids; and (3) exclusion of infection from the results of sputum culture, and serum antigens or antibodies for specific microorganisms or BAL. Drug-induced pneumonia could progress despite steroid administration especially with DAD pattern; however, the patients with progression were not included in this study because it could be difficult to specify the cause of diffuse GGA and exclude other noninfectious or infectious complications.

DAH: (1) clinical symptoms (massive hemoptysis) under the state of thrombocytopenia.

Pulmonary edema: (1) the presence of heart failure confirmed by echocardiography; and (2) an immediate response to diuretics and/or cardiotonic agents.

IPS: (1) clinically defined by widespread lung injury after HSCT; (2) the absence of infection; and (3) the absence of cardiac, renal or iatrogenic etiology [5].

The subjects recruited for this study were previously reported in other studies [6,7]. One study included 345 subjects and compared HRCT findings among infectious diseases [6], and the other study included 555 subjects and compared HRCT findings between infectious and noninfectious diseases [7]. The goal of the present study was to compare the findings of patients with extensive GGA on HRCT; thus, the purpose of the current study differs from that of the previous reports $[6,7]$.

\section{CT examinations}

CT scans were obtained using a TCT-900S (Canon Medical Systems Corporations), a SOMATOM PLUS 4, a Volume Zoom, Somatom Definition, or Somatom Sensation 64 (Siemens). CT scans were obtained at suspended end-inspiratory 


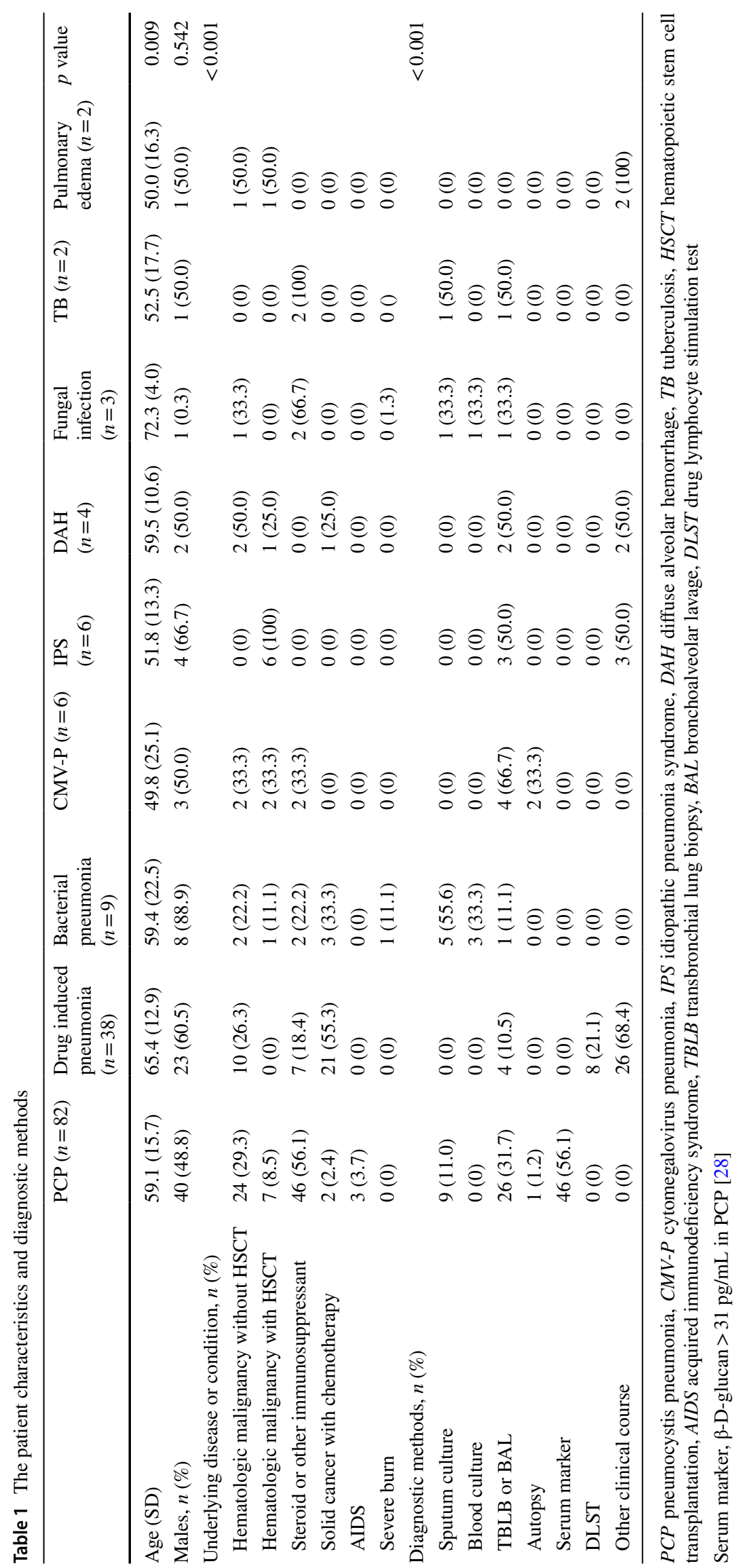


effort in the supine position, basically without intravenous contrast material. With a TCT-900S scanner, after 10-mm collimation scans were obtained in contiguous 10 -mm intervals through the entire chest, all patients underwent HRCT through the region showing abnormal parenchymal findings at $2 \mathrm{~mm}$ collimation. With other multislice CT scanners, after contiguous 5-, 7- or 10-mm section CT was performed through the chest, additional HRCT images, consisting of 1- or 2-mm collimated images were obtained at 1-, 2-, 5-, or 10 -mm intervals through the abnormal lung parenchyma. In all patients, the scanning parameters were $120-140 \mathrm{kVp}$ and 160-250 effective mAs.

While image data were viewed on hard copy films when CT was performed with a TCT-900S, all image data were interfaced directly to our picture archiving and communication system (PACS) (ShadeQuest; Yokogawa Medical Solutions Corp.), which displayed all image data on monitors (three monitors, $1280 \times 1080$ matrix, 8-bit viewable grayscale) when CT was performed using other multislice CT scanners. The monitors were used to view both lung (window width, 1500-1750 HU; window level, -600 to -700 $\mathrm{HU}$ ) and mediastinal (window width, 250-400 HU; window level, 40-50 HU) window images.

\section{Evaluation of CT images}

The CT images were assessed independently by two boardcertified chest radiologists (15 and 28 years of experience, respectively) who had no knowledge of the patients' clinical information other than the fact that all patients were immunocompromised hosts. Discordant results between the two radiologists were resolved by consensus of the same two radiologists.

The presence or absence of the following HRCT findings were coded: (a) crazy-paving pattern, (b) mosaic pattern (mosaic perfusion), (c) airspace consolidation, (d) nodules (e) bronchovascular bundle (BVB) thickening, (f) interlobular septal (ILS) thickening, (g) hilar or mediastinal lymph node (LN) swelling, and (h) pleural effusion.

The presence of GGA was defined if there was a hazy increase in lung opacity without obscuration of vessels. The presence of crazy-paving pattern was recorded if there was superimposition of interlobular or intralobular interstitial thickening within the GGA. Mosaic pattern was defined as sharply demarcated lung areas of inhomogeneous attenuation in which intervening areas of the normal lung were observed between GGAs.

The presence of airspace consolidation was defined if there was increased lung attenuation with obscuration of vessels.

Nodules were classified in size as micro $(<3 \mathrm{~mm})$, small $(>3$ to $<10 \mathrm{~mm})$, or large $(\geq 10 \mathrm{~mm})$, and in distribution as centrilobular, perilymphatic or random. Centrilobular distribution was defined by the presence of nodules around the peripheral pulmonary bronchial branches or 3-5 mm from the pleura, interlobular septa, pulmonary veins, or relatively proximal pulmonary arteries. Perilymphatic distribution was defined by the presence of nodules around the peribronchovascular interstitium, interlobular septa, and subpleural regions. Random distribution was defined when nodules did not show any specific distribution within the secondary pulmonary lobules.

The overall lesional distribution was classified axially; overall axial distribution was defined as central, peripheral, diffuse or indeterminate and craniocaudal; overall craniocaudal distribution was defined as upper, lower, diffuse or indeterminate.

\section{Statistical analysis}

Interobserver agreement between the 2 radiologists was assessed by calculating the kappa value ( $\kappa$ value) for the above-mentioned HRCT findings from (a) to (j): poor $(\kappa=0.00-0.20)$, fair $(\kappa=0.21-0.40)$, moderate $(\kappa=0.41-0.60)$, good $(\kappa=0.61-0.80)$, or excellent $(\kappa=0.81-1.00)[8]$.

Each CT finding and CT pattern was compared between the diseases using a chi-squared $\left(\chi^{2}\right)$ test for independence. Age was compared using the Kruskal Wallis test.

A multiple logistic regression analysis was performed in order to identify significant indicators for differentiation among diseases, for example, between PCP and nonPCP (a combination of drug-induced pneumonia, bacterial pneumonia, CMV-P, IPS, DAH, fungal infection, TB, and pulmonary edema), and between drug-induced pneumonia and non-drug induced pneumonia (a combination of PCP, bacterial pneumonia, CMV-P, IPS, DAH, fungal infection, $\mathrm{TB}$, and pulmonary edema). The forward selection method was used for the multiple logistic regression analysis, and independent variables included all HRCT findings (12 criteria). The area under the curve (AUC) of each model was calculated. All statistical analyses were performed using a commercially available software program (SPSS, version 22.0, IBM). $P$ values of $<0.05$ were considered to indicate statistical significance.

\section{Results}

Table 1 shows the characteristics of patients and the diagnostic methods. Age, underlying disease or condition, and diagnostic methods were significantly different among the groups $(p<0.05)$. Table 2 shows the analysis of HRCT findings of pulmonary complications using the $\chi^{2}$ test. The interobserver agreement was $0.374-0.775$ (fair-good). There were significant differences in consolidation $(p=0.041)$, 


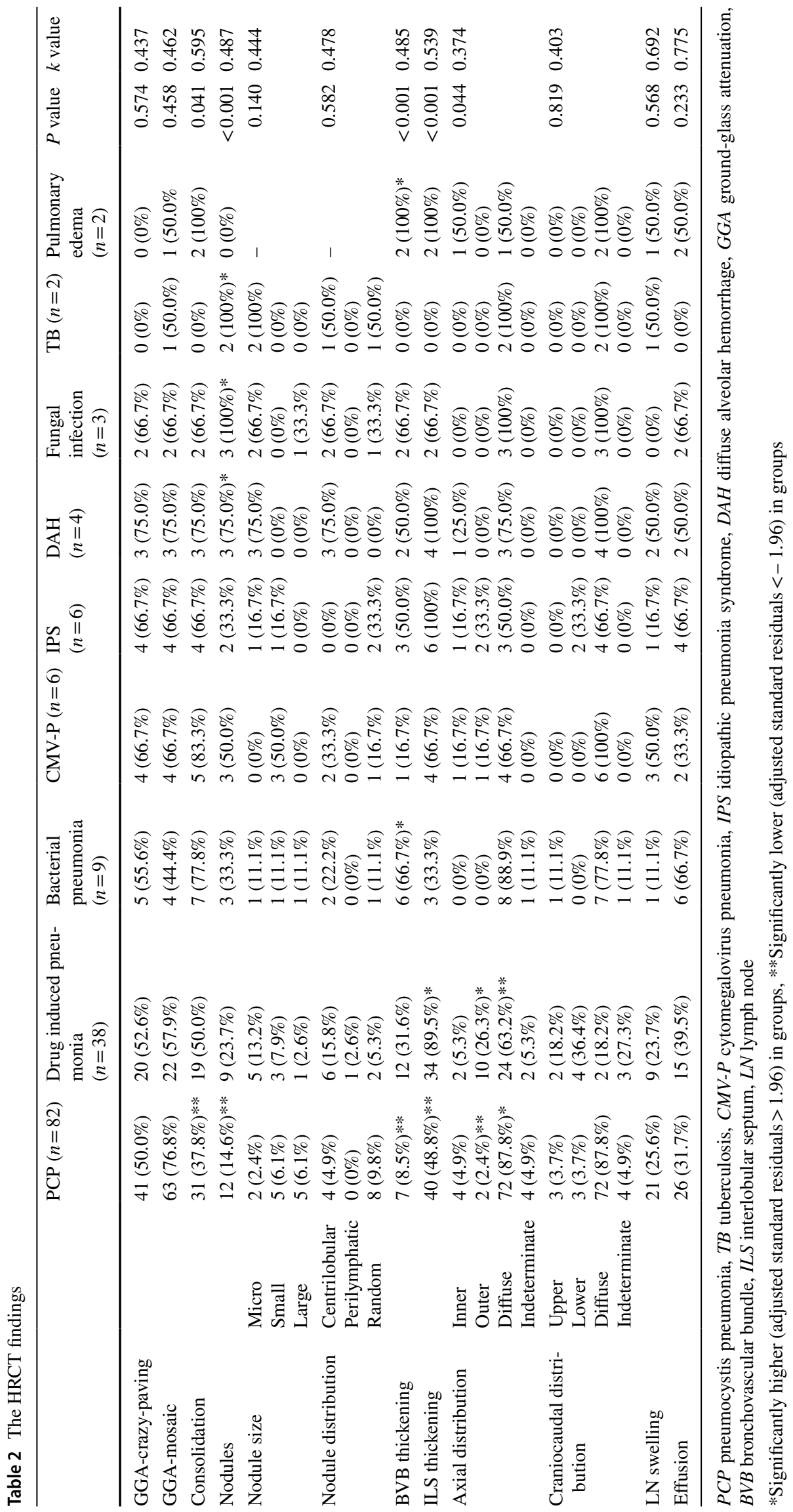




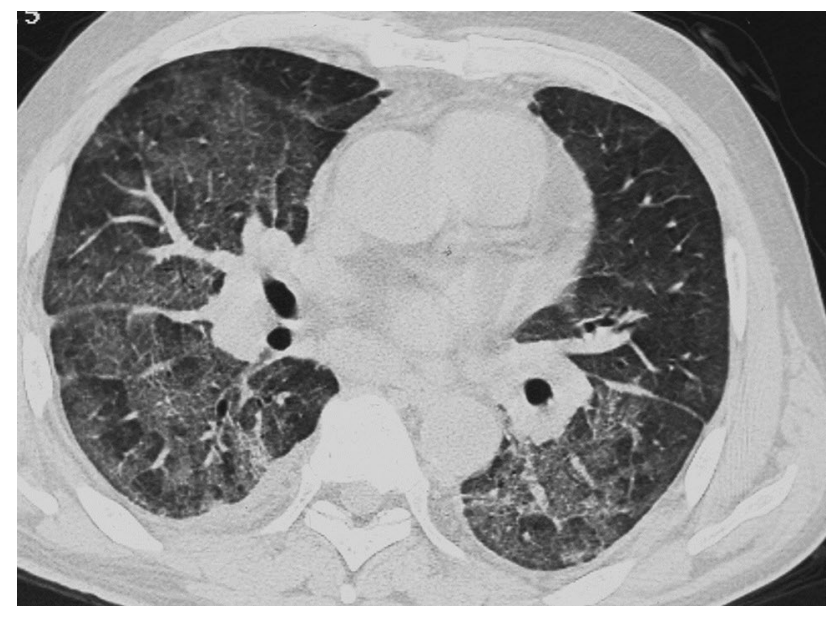

Fig. 1 A 60-year-old man with pneumocystis pneumonia under treatment for acute lymphoid leukemia. High-resolution computed tomography shows extensive ground-glass attenuation with a mosaic pattern

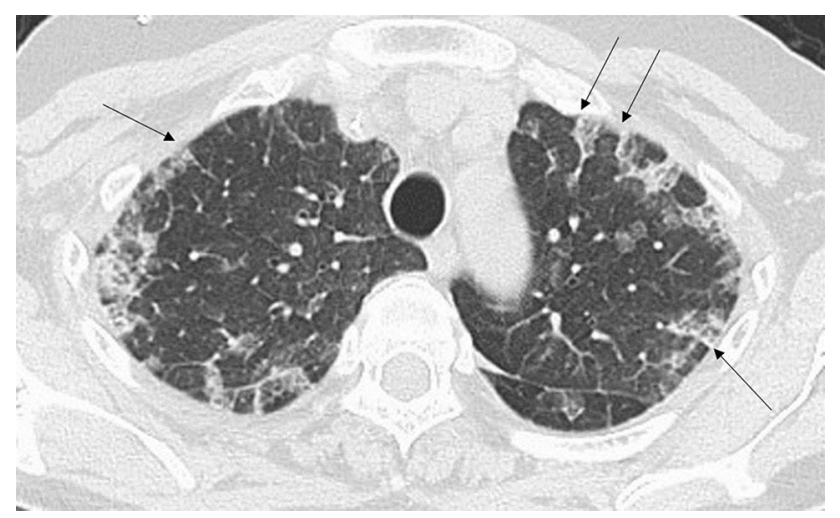

Fig. 2 A 61-year-old woman with drug-induced pneumonia under chemotherapy for colon cancer. High-resolution computed tomography shows ground-glass attenuation with outer distribution and thickening of the interlobular septum (arrows)

nodule ( $p<0.001)$, BVB thickening $(p<0.001)$, ILS thickening $(p<0.001)$, and axial distribution $(p=0.044)$ among the groups. For PCP, the frequencies of consolidation (37.8\%), nodule $(14.6 \%)$, BVB thickening $(8.5 \%)$, ILS thickening $(48.8 \%)$, and outer distribution (2.4\%) were significantly low, and the frequency of diffuse axial distribution (87.8\%) was significantly high among the groups (Fig. 1). For druginduced pneumonia, the frequencies of ILS thickening $(89.5 \%)$ and outer distribution $(26.3 \%)$ were significantly high (Fig. 2) and the frequency of diffuse axial distribution $(63.2 \%)$ was significantly low among the groups. The ILS thickening was also frequent in other noninfectious disease; IPS, DAH, and pulmonary edema (100\%, respectively) and in infectious disease; CMV-P and fungal infection $(66.7 \%$, respectively) (Table 2). The frequency of BVB thickening was significantly high among patients with bacterial

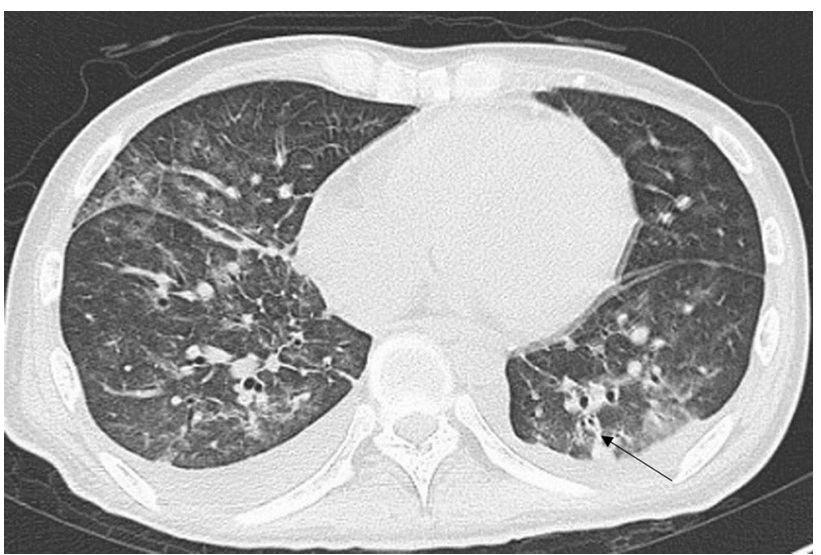

Fig. 3 A 29-year-old man with bacterial pneumonia under treatment for acute leukemia. High-resolution computed tomography shows patchy ground-glass attenuation with bronchovascular bundle thickening (arrow)

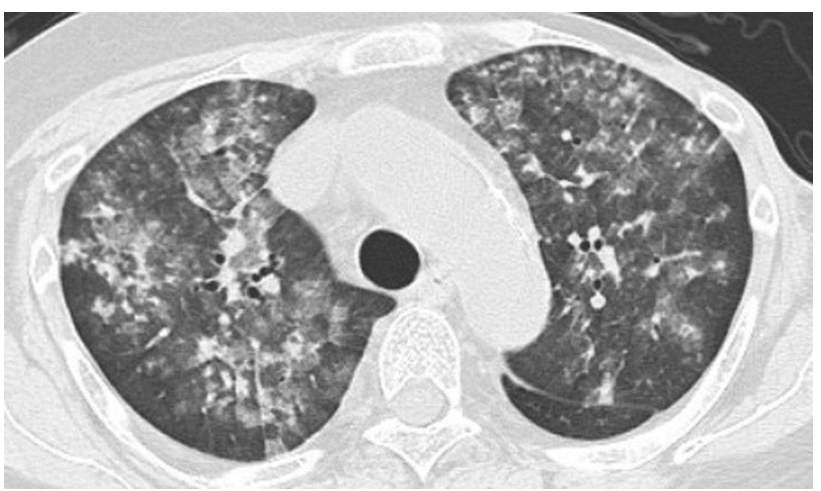

Fig. 4 A 56-year-old woman with diffuse alveolar hemorrhage under treatment for thrombocytopenia and uterine cancer. High-resolution computed tomography shows extensive ground-glass attenuation with multiple nodular lesions

pneumonia (66.7\%) (Fig. 3) and those with pulmonary edema (100\%). The frequency of nodules was significantly high among patients with DAH (75.0\%) (Fig. 4), fungal infection (100\%), and TB (100\%).

The multiple logistic regression analyses identified significant indicators for each disease (Table 3 ). The absence of nodules ( $p=0.015$; odds ratio [OR], 2.990; 95\% confidence interval [CI], 1.239-7.214; sensitivity, 85.4\%; specificity, $35.7 \%)$, the absence of BVB thickening ( $p=0.001$; OR, 5.158; 95\% CI, 1.983-13.414; sensitivity, $91.5 \%$; specificity, $40.0 \%$ ), and the absence of ILS thickening ( $p=0.002$; OR, 3.322 ; 95\% CI, 1.527-7.228; sensitivity, 51.2\%; specificity, $78.6 \%$ ) were identified as indicators of PCP (Tables 3 and 4). The presence of ILS thickening ( $p<0.001$; OR, 7.385; 95\% CI, 2.460-22.174; sensitivity, $89.5 \%$; specificity, $46.5 \%$ ) was identified as an indicator of drug-induced pneumonia (Tables 3 and 4). The presence of BVB thickening 
Table 3 The results of the multiple logistic regression analysis

\begin{tabular}{|c|c|c|c|c|c|c|}
\hline \multicolumn{2}{|l|}{ HRCT findings } & \multirow{2}{*}{$\frac{\mathrm{PCP}(n=82)}{12(14.6 \%)}$} & \multirow{2}{*}{$\begin{array}{l}\text { Non-PCP }(n=70) \\
25(35.7 \%)\end{array}$} & \multirow{2}{*}{$\begin{array}{l}\text { Wald } \\
5.940\end{array}$} & \multirow{2}{*}{$\begin{array}{l}\text { Odds ratio }[95 \% \mathrm{CI}] \\
-\end{array}$} & \multirow{2}{*}{$\begin{array}{c}p \text { value } \\
0.015\end{array}$} \\
\hline Nodule & Positive & & & & & \\
\hline & Negative & $70(85.4 \%)$ & $45(64.3 \%)$ & & $2.990[1.239,7.214]$ & \\
\hline BVB thickening & Positive & $7(8.5 \%)$ & $28(40.0 \%)$ & 11.316 & - & 0.001 \\
\hline & Negative & $75(91.5 \%)$ & $42(60.0 \%)$ & & $5.158[1.983,13.414]$ & \\
\hline \multirow[t]{3}{*}{ ILS thickening } & Positive & $40(48.8 \%)$ & $55(78.6 \%)$ & 9.162 & - & 0.002 \\
\hline & Negative & $42(51.2 \%)$ & $15(21.4 \%)$ & & $3.322[1.527,7.228]$ & \\
\hline & & $\begin{array}{l}\text { Drug induced pneumonia } \\
(n=38)\end{array}$ & $\begin{array}{l}\text { Non-drug induced pneumonia } \\
(n=114)\end{array}$ & Wald & Odds ratio $[95 \% \mathrm{CI}]$ & $p$ value \\
\hline \multirow[t]{3}{*}{ ILS thickening } & Positive & $34(89.5 \%)$ & $61(48.3 \%)$ & 12.705 & $7.385[2.460,22.174]$ & $<0.001$ \\
\hline & Negative & $4(10.5 \%)$ & $53(46.5 \%)$ & & - & \\
\hline & & Bacterial pneumonia $(n=9)$ & $\begin{array}{l}\text { Non-bacterial pneumonia } \\
(n=143)\end{array}$ & Wald value & Odds ratio $[95 \% \mathrm{CI}]$ & $p$ value \\
\hline \multirow[t]{3}{*}{ BVB thickening } & Positive & $6(66.7 \%)$ & $29(20.3 \%)$ & 7.827 & $7.862[1.854,33.337]$ & 0.005 \\
\hline & Negative & $3(33.3 \%)$ & $114(79.7 \%)$ & & - & \\
\hline & & $\mathrm{DAH}(n=4)$ & Non-DAH $(n=148)$ & Wald & Odds ratio $[95 \% \mathrm{CI}]$ & $p$ value \\
\hline \multirow[t]{2}{*}{ Nodule } & Positive & $3(75.0 \%)$ & $34(23.0 \%)$ & 3.885 & $10.059[1.013,99.863]$ & 0.049 \\
\hline & Negative & $1(25.0 \%)$ & $114(77.0 \%)$ & & - & \\
\hline
\end{tabular}

$P C P$ pneumocystis pneumonia, $B V B$ bronchovascular bundle, $I L S$ interlobular septum, $D A H$ diffuse alveolar hemorrhage, $C I$ confidence interval

Table 4 Sensitivity, specificity, accuracy, PPV, and NPV of each HRCT finding for detecting each infection

\begin{tabular}{llllll}
\hline & Sensitivity & Specificity & PPV & NPV & AUC \\
\hline PCP & & & & & \\
Absence of nodules & $85.4 \%$ & $35.7 \%$ & $60.9 \%$ & $67.6 \%$ & 0.605 \\
Absence of BVB thickening & $91.5 \%$ & $40.0 \%$ & $64.1 \%$ & $80.0 \%$ & 0.657 \\
$\quad$ Absence of ILS thickening & $51.2 \%$ & $78.6 \%$ & $73.7 \%$ & $57.9 \%$ & 0.649 \\
$\begin{array}{l}\text { Drug induced pneumonia } \\
\quad \text { Presence of ILS thickening }\end{array}$ & $89.5 \%$ & $46.5 \%$ & $35.8 \%$ & $93.0 \%$ & 0.680 \\
Bacterial pneumonia & & & & \\
$\quad$ Presence of BVB thickening & $66.7 \%$ & $79.7 \%$ & $17.1 \%$ & $97.4 \%$ & 0.732 \\
DAH & & & & & \\
$\quad$ Presence of nodules & $75.0 \%$ & $77.0 \%$ & $8.1 \%$ & $99.1 \%$ & 0.760 \\
\hline
\end{tabular}

$P P V$ positive predictive value, $N P V$ negative predictive value, $P C P$ pneumocystis pneumonia, $B V B$ bronchovascular bundle, ILS interlobular septum, $D A H$ diffuse alveolar hemorrhage, $A U C$ area under the curve
( $p=0.005$; OR, 10.059; 95\% CI, 1.013-99.863; sensitivity, $66.7 \%$; specificity, $79.7 \%$ ) was identified as an indicator of bacterial pneumonia (Tables 3 and 4 ). The presence of nodules ( $p=0.049$; OR, 10.059; 95\% CI, 1.013-99.863; sensitivity, $75.0 \%$; specificity, $77.0 \%$ ) was identified as an indicator of DAH (Tables 3 and 4). The AUC values were $0.605-0.760$.

\section{Discussion}

Our study suggested that differentiation between pulmonary complications with acute or subacute extensive GGA in immunocompromised patients might be possible by evaluating various HRCT findings. Extensive GGA often causes difficulty in making a clinical diagnosis, especially in immunocompromised patients; however, our results may be useful for an early and correct diagnosis and for selecting appropriate treatment.

In patients with PCP, the frequencies of consolidation, nodules, BVB thickening, ILS thickening, and outer distribution were significantly low and the frequency of diffuse axial distribution was significantly high in comparison to the other groups in our study. In particular, the absence of nodules and the absence of BVB thickening could be indicators for differentiation. In PCP, nodules have been reported as infrequent findings $[9,10]$, which may support the results of the present study. 
In patients with drug-induced pneumonia, the frequencies of ILS thickening and outer distribution were significantly high in comparison to the other groups in our study. ILS thickening is seen in lymphatic or infiltrative disease. It is frequently observed in drug-induced pneumonia [11]; however, it is also a common finding in pulmonary edema and was observed in 2 patients with pulmonary edema in this study $(100 \%)$. In addition, the frequency of ILS thickening was high in other noninfectious diseases including IPS and DAH and infectious diseases including CMV-P and fungal infection in this study (66.7-100\%), with a small number of subjects. Thus, it may be a non-specific finding among pulmonary complications with extensive GGA.

Bilateral diffuse opacities can be observed in immunocompromised patients with bacterial pneumonia [12]. In patients with bacterial pneumonia, the frequency of BVB thickening was significantly high and BVB thickening could be used as an indicator in our study. The BVB is the connective tissue sheath that encloses the bronchi and hilar vessels; thus, BVB thickening includes thickening of the bronchial wall and bronchovascular interstitium [13]. Several studies reported that bronchial wall thickening due to inflammatory reactions was frequently observed in patients with bacterial pneumonia [14-16]. The frequency was also relatively higher in patients with a fungal infection, IPS, and DAH; thus, it could be a nonspecific finding among the pulmonary complications with extensive GGA.

Among the groups in our study, nodules were significantly frequent in the DAH, fungal infection, and TB groups. It may be an indicator in DAH. Diffuse nodular opacities and patchy GGA have been reported to be the most common findings in DAH and nodules are observed due to deposition of hemosiderin-laden macrophages in alveolar spaces and small vessels, or capillaritis $[17,18]$. In TB, nodules are a frequent $\mathrm{CT}$ finding in both immunocompetent and immunocompromised patients [19]. In fungal infections, including invasive aspergillosis and infections other than aspergillosis, the predominant CT findings include nodules [20-23]. These reports support our study. However, according to our results, when small nodules are observed coexisting with extensive GGA, the possibility of DAH should be considered.

There were no significant differences in CMV-P or IPS among the groups in our study. HRCT findings of CMV-P have been described as being a mixture of patterns with GGA, consolidation, and small nodules [24, 25]. Our study also supported the findings of the previous studies and these various findings could make it difficult to diagnose CMV-P in patients with extensive GGA. CMV-P and PCP are often difficult to distinguish because of the clinical and radiological similarities. HRCT findings for the differential diagnosis of CMV-P and PCP included extensive GGA with a mosaic pattern and an apical distribution in PCP, and an ill-defined demarcation of GGA, consolidation, and nodules in CMV-P [26, 27]. IPS included a variety of clinical, histological, and radiographic patterns [4, 5]. This could cause difficulty in the diagnosis of IPS among patients with extensive GGA.

Our study was associated with several limitations. First, the present study was retrospective in nature; thus, the CT protocols and diagnostic procedures were diverse. Second, the number of cases of diseases other than PCP and druginduced pneumonia was relatively small. The number of HIV-infected patients was also small due to the single-center nature of the study. This may have affected the CT findings especially in PCP patients. The patients with viral pneumonia other than CMV-P were not included in this study. The recent SARS-COV-2 infection was not evaluated in this study though it is frequent in the present and could continue in the future. Third, the reliability of the diagnosis may be controversial because pathological confirmation and the resultant radiologic-pathologic correlation was only available for a small number of cases. However, it is often difficult for immunocompromised patients to undergo invasive procedures. More than one disease can coexist in extensive GGA in clinical practice; however, our study population was limited to patients with only one final diagnosis. Therefore, there is no guarantee that the results of this study always apply to clinical cases.

In conclusion, differentiation between pulmonary complications with extensive GGA in immunocompromised patients may be possible using HRCT findings. Nodules, BVB thickening, and ILS thickening were found to be particularly useful HRCT findings.

\section{Declarations}

Conflict of interest The authors have no conflict of interest to disclose with respect to this manuscript.

Ethical approval The authors declare that they preserve ethical standards.

Open Access This article is licensed under a Creative Commons Attribution 4.0 International License, which permits use, sharing, adaptation, distribution and reproduction in any medium or format, as long as you give appropriate credit to the original author(s) and the source, provide a link to the Creative Commons licence, and indicate if changes were made. The images or other third party material in this article are included in the article's Creative Commons licence, unless indicated otherwise in a credit line to the material. If material is not included in the article's Creative Commons licence and your intended use is not permitted by statutory regulation or exceeds the permitted use, you will need to obtain permission directly from the copyright holder. To view a copy of this licence, visit http://creativecommons.org/licenses/by/4.0/. 


\section{References}

1. Engeler CE, Tashjian JH, Trenkner SW, Walsh JW. Ground-glass opacity of the lung parenchyma: a guide to analysis with highresolution CT. AJR Am J Roentgenol. 1993;160(2):249-51.

2. Leung AN, Miller RR, Muller NL. Parenchymal opacification in chronic infiltrative lung diseases: CT-pathologic correlation. Radiology. 1993;188(1):209-14.

3. Miller WT Jr, Shah RM. Isolated diffuse ground-glass opacity in thoracic CT: causes and clinical presentations. AJR Am J Roentgenol. 2005;184(2):613-22.

4. Tanaka N, Kunihiro Y, Yujiri T, Ando T, Gondo T, Kido S, et al. High-resolution computed tomography of chest complications in patients treated with hematopoietic stem cell transplantation. Jpn J Radiol. 2011;29(4):229-35.

5. Panoskaltsis-Mortari A, Griese M, Madtes DK, Belperio JA, Haddad IY, Folz RJ, et al. An official American Thoracic Society research statement: noninfectious lung injury after hematopoietic stem cell transplantation: idiopathic pneumonia syndrome. Am J Respir Crit Care Med. 2011;183(9):1262-79.

6. Kunihiro Y, Tanaka N, Kawano R, Yujiri T, Kubo M, Ueda K, et al. Differential diagnosis of pulmonary infections in immunocompromised patients using high-resolution computed tomography. Eur Radiol. 2019;29(11):6089-99.

7. Tanaka N, Kunihiro Y, Kawano R, Yujiri T, Ueda K, Gondo T, et al. Chest complications in immunocompromised patients without acquired immunodeficiency syndrome (AIDS): differentiation between infectious and non-infectious diseases using high-resolution CT findings. Clin Radiol. 2021;76(1):50-9.

8. Maclure M, Willett WC. Misinterpretation and misuse of the kappa statistic. Am J Epidemiol. 1987;126(2):161-9.

9. Boiselle PM, Crans CA Jr, Kaplan MA. The changing face of Pneumocystis carinii pneumonia in AIDS patients. AJR Am J Roentgenol. 1999;172(5):1301-9.

10. Kuhlman JE, Kavuru M, Fishman EK, Siegelman SS. Pneumocystis carinii pneumonia: spectrum of parenchymal CT findings. Radiology. 1990;175(3):711-4.

11. Cleverley JR, Screaton NJ, Hiorns MP, Flint JD, Muller NL. Druginduced lung disease: high-resolution CT and histological findings. Clin Radiol. 2002;57(4):292-9.

12. Franquet T. High-resolution computed tomography (HRCT) of lung infections in non-AIDS immunocompromised patients. Eur Radiol. 2006;16(3):707-18.

13. Tuddenham WJ. Glossary of terms for thoracic radiology: recommendations of the Nomenclature Committee of the Fleischner Society. AJR Am J Roentgenol. 1984;143(3):509-17.

14. Morikawa K, Okada F, Ando Y, Ishii R, Matsushita S, Ono A, et al. Meticillin-resistant Staphylococcus aureus and meticillinsusceptible $\mathrm{S}$. aureus pneumonia: comparison of clinical and thinsection CT findings. Br J Radiol. 2012;85(1014):e168-75.

15. Okada F, Ando Y, Nakayama T, Tanoue S, Ishii R, Ono A, et al. Pulmonary thin-section CT findings in acute Moraxella catarrhalis pulmonary infection. Br J Radiol. 2011;84(1008):1109-14.

16. Okada F, Ando Y, Tanoue S, Ishii R, Matsushita S, Ono A, et al. Radiological findings in acute Haemophilus influenzae pulmonary infection. Br J Radiol. 2012;85(1010):121-6.
17. Cheah FK, Sheppard MN, Hansell DM. Computed tomography of diffuse pulmonary haemorrhage with pathological correlation. Clin Radiol. 1993;48(2):89-93.

18. Primack SL, Miller RR, Müller NL. Diffuse pulmonary hemorrhage: clinical, pathologic, and imaging features. AJR Am J Roentgenol. 1995;164(2):295-300.

19. Ikezoe J, Takeuchi N, Johkoh T, Kohno N, Tomiyama N, Kozuka T, et al. CT appearance of pulmonary tuberculosis in diabetic and immunocompromised patients: comparison with patients who had no underlying disease. AJR Am J Roentgenol. 1992;159(6):1175-9.

20. Chang WC, Tzao C, Hsu HH, Lee SC, Huang KL, Tung HJ, et al. Pulmonary cryptococcosis: comparison of clinical and radiographic characteristics in immunocompetent and immunocompromised patients. Chest. 2006;129(2):333-40.

21. Franquet T, Muller NL, Lee KS, Oikonomou A, Flint JD. Pulmonary candidiasis after hematopoietic stem cell transplantation: thin-section CT findings. Radiology. 2005;236(1):332-7.

22. Kuhlman JE, Fishman EK, Burch PA, Karp JE, Zerhouni EA, Siegelman SS. Invasive pulmonary aspergillosis in acute leukemia. The contribution of CT to early diagnosis and aggressive management. Chest. 1987;92(1):95-9.

23. McAdams HP, de Christenson MR, Strollo DC, Patz EF Jr. Pulmonary mucormycosis: radiologic findings in 32 cases. AJR Am J Roentgenol. 1997;168(6):1541-8.

24. Franquet T, Lee KS, Muller NL. Thin-section CT findings in 32 immunocompromised patients with cytomegalovirus pneumonia who do not have AIDS. AJR Am J Roentgenol. 2003;181(4):1059-63.

25. Gasparetto EL, Ono SE, Escuissato D, Marchiori E, Roldan L, Marques HL, et al. Cytomegalovirus pneumonia after bone marrow transplantation: high resolution CT findings. Br J Radiol. 2004;77(921):724-7.

26. Kunihiro Y, Tanaka N, Matsumoto T, Yamamoto N, Matsunaga N. The usefulness of a diagnostic method combining high-resolution CT findings and serum markers for cytomegalovirus pneumonia and pneumocystis pneumonia in non-AIDS patients. Acta Radiol. 2015;56(7):806-13.

27. Vogel MN, Brodoefel H, Hierl T, Beck R, Bethge WA, Claussen $\mathrm{CD}$, et al. Differences and similarities of cytomegalovirus and pneumocystis pneumonia in HIV-negative immunocompromised patients thin section CT morphology in the early phase of the disease. Br J Radiol. 2007;80(955):516-23.

28. Tasaka S, Hasegawa N, Kobayashi S, Yamada W, Nishimura T, Takeuchi T, et al. Serum indicators for the diagnosis of pneumocystis pneumonia. Chest. 2007;131:1173-80.

Publisher's Note Springer Nature remains neutral with regard to jurisdictional claims in published maps and institutional affiliations. 